\title{
XMM-Newton spectral properties of the ultraluminous IRAS galaxy Mrk 273
}

\author{
I. Balestra, Th. Boller, L. Gallo, D. Lutz, and S. Hess
}

Max-Planck-Institut für extraterrestrische Physik, Postfach 1312, 85741 Garching, Germany

e-mail: balestra@mpe.mpg.de

Received 2 June 2005 / Accepted 12 July 2005

\begin{abstract}
We present a 23 ks XMM-Newton observation of the Ultraluminous Infrared Galaxy (ULIRG) Mrk 273. The hard X-ray spectrum can be modeled by a highly absorbed $\left(\sim 7 \times 10^{23} \mathrm{~cm}^{-2}\right)$ power law plus an Fe K $\alpha$ emission line. The iron line (detected at more than $99 \%$ c.1.) is broad $\left(\sigma=0.26_{-0.17}^{+0.37} \mathrm{keV}\right)$, suggesting possible superposition of a neutral iron line at $6.4 \mathrm{keV}$, and a blend of ionized iron lines from Fe XXV and Fe XXVI. Given the relatively short exposure, the three line components cannot be individually resolved with high statistical significance: the neutral component is detected at $\sim 2.5 \sigma$ and the Fe XXV line at $\sim 2 \sigma$ c.l., while for the Fe XXVI line we can only estimate an upper limit. The broad band spectrum requires, in addition to a highly absorbed power law, at least three collisionally ionized plasma components, which may be associated with star-forming regions. The temperatures of the three plasmas are about $0.3,0.8$ and $6 \mathrm{keV}$, where the highest of the three is sufficient to produce ionized iron emission lines. An alternative interpretation for the origin of the soft emission might also be reflection off photoionized gas, as has been observed in a number of nearby Compton-thick Seyfert 2 galaxies (e.g. NGC 1068, Circinus, Mrk 3, NGC 4945). A hot gas, photoionized by the primary, continuum can also produce ionized iron lines. Unfortunately, given the limited statistics and the lack of high resolution spectroscopy, it is not possible to distinguish between the two models investigated. We further compare the XMM-Newton findings with the Chandra data obtaining consistent spectral results. The absorption-corrected hard X-ray luminosity of Mrk 273 is $L_{2-10 \mathrm{keV}} \sim 7 \times 10^{42} \mathrm{erg} \mathrm{s}^{-1}$, corresponding to $\sim 0.2 \%$ of the far-IR luminosity, similar to typical values found in pure starbursts. The thermal contribution to the soft X-ray luminosity is approximately $0.2-0.7 \times 10^{42} \mathrm{erg} \mathrm{s}^{-1}$, comparable to those found in NGC 6240 and other starburst-dominated ULIRGs. We also analyze the XMM-Newton spectrum of Mrk 273x, an unabsorbed Seyfert 2 galaxy at redshift $z=0.458$, which lies in the field of view of Mrk 273.
\end{abstract}

Key words. galaxies: individual: Mrk 273 - galaxies: individual: Mrk 273x - galaxies: Seyfert - X-rays: galaxies

\section{Introduction}

The InfraRed Astronomical Satellite (IRAS) has detected a very large number of galaxies in the local universe $(z<0.3)$ which exhibit extraordinarily high infrared luminosity $\left(L_{\mathrm{IR}} \geq\right.$ $10^{12} L_{\odot}$ for $H_{0}=75 \mathrm{~km} \mathrm{~s}^{-1} \mathrm{Mpc}^{-1}$; see Sanders \& Mirabel 1996, for a review). The number density of these so called Ultraluminous InfraRed Galaxies (ULIRGs) exceeds that of optically selected Seyfert galaxies and QSOs with comparable bolometric luminosities (Soifer et al. 1987; Sanders et al. 1988a,b; Sanders \& Mirabel 1996) by a factor of $\sim 1.5-2$ (Sanders et al. 1999). The bulk of luminosity in these sources is infrared (IR) emission from warm dust. Only two dust-heating mechanisms are capable of producing such an extraordinary IR luminosity: one involves the presence of a strong starburst region, and the other a dust-enshrouded AGN. However it is still not clear what the relative contribution of each component to their bolometric luminosity should be.

Spectroscopic surveys of samples of ULIRGs carried out with the Infrared Space Observatory (ISO) have revealed that about $80 \%$ of such objects are predominantly powered by star formation, but the fraction of AGN-powered objects increases with luminosity (Genzel et al. 1998; Lutz et al. 1998).

X-ray observations are a fundamental tool to probe the highly obscured innermost regions of these objects, and therefore potentially unveil the physical processes at work. In fact a considerable portion of ULIRGs has been found to contain a hard X-ray source, highly absorbed by a molecular torus, which indicates the presence of a hidden AGN (Mitsuda 1995; Brandt et al. 1997; Kii et al. 1997; Vignati et al. 1999; Ptak et al. 2003; Braito et al. 2003; Franceschini et al. 2003).

Mrk 273 is a well studied ULIRG at a redshift $z=0.03778$ with a Seyfert 2 nucleus (Koski 1978; Sanders et al. 1988a). It presents evidences for strong star formation (Goldader et al. 1995), such as strong Polycylic Aromatic Hydrocarbons (PAH) features in the near-IR (Genzel et al. 1998) and extended $(\sim 370 \mathrm{pc})$ radio emission, punctuated by a number of compact sources identifiable as supernovae or supernova remnants (Carilli \& Taylor 2000; Bondi et al. 2005). Like many ULIRGs, Mrk 273 presents clear indications of an ongoing merging 
process such as a long tidal tail and a double nucleus (Knapen et al. 1997; Soifer et al. 2000; Carilli \& Taylor 2000).

ASCA X-ray observations of Mrk 273 showed evidence of a highly absorbed $\left(\sim 4 \times 10^{23} \mathrm{~cm}^{-2}\right)$ hard component above about $3 \mathrm{keV}$ together with soft thermal emission (Turner et al. 1997, 1998; Iwasawa 1999). These findings were indeed confirmed by Chandra, which revealed a compact hard X-ray nucleus inside a much more extended soft halo (Xia et al. 2002, hereafter Paper I).

In this paper we will discuss the analysis of the XMM-Newton data of Mrk 273 and compare them with the previous Chandra observation (Paper I). The data analysis and details of the XMM-Newton observation are described in Sect. 2, together with our updated reduction of the Chandra data. In Sect. 3 the spectral fitting results are presented. A comparison with the Chandra observation is given in Sect. 4. Our results are then discussed in Sect. 5 and a summary of the XMMNewton results on Mrk 273 is given in Sect. 6. In Appendix A we discuss the XMM-Newton spectrum of Mrk 273x, an unabsorbed Seyfert 2 observed serendipitously in the field of view of Mrk 273.

Throughout this paper we use the following cosmological parameters: $H_{0}=70 \mathrm{~km} \mathrm{~s}^{-1} \mathrm{Mpc}^{-1}, \Lambda_{0}=0.7$ and $q_{0}=0$. Within the adopted cosmology $1^{\prime \prime}$ corresponds to $0.75 \mathrm{kpc}$ at the redshift of Mrk 273.

\section{Observations and data reduction}

\subsection{XMM-Newton}

Mrk 273 was observed with XMM-Newton (Jansen et al. 2001) for $23 \mathrm{ks}$ on 2002 May 07 (revolution 0441). During this time the EPIC-PN (Struider et al. 2001) and MOS (MOS1 and MOS2; Turner et al. 2001) cameras, as well as the Optical Monitor (OM; Mason et al. 2001) and the Reflection Grating Spectrometers (RGS1 and RGS2; den Herder et al. 2001) collected data. The EPIC-PN and MOS cameras were operated in full-frame mode and utilized the thick filter.

The Observation Data Files were processed to produce calibrated event lists using the XMM-Newton Science Analysis System (SAS v6.1.0). Unwanted hot, dead, or flickering pixels were removed as were events due to electronic noise. Event energies were corrected for charge-transfer losses, and EPIC response matrices were generated using the SAS tasks ARFGEN and RMFGEN. Light curves were extracted from these event lists to search for periods of high background flaring. Background flaring was negligible. The total good exposure times selected for the PN and MOS were 18 and $22 \mathrm{ks}$, respectively.

The source plus background photons were extracted from a circular region with a radius of 50", and the background was selected from an off-source region with a radius of $70^{\prime \prime}$ and appropriately scaled to the source region. Single and double events were selected for the PN detector, and single-quadruple events were selected for the MOS. The resulting PHA files were grouped with a minimum of 20 counts per bin. Pile-up effects were determined to be negligible.

The RGS were operated in standard Spectro+Q mode. The first-order RGS spectra were extracted using the SAS task
RGSPROC, and the response matrices were generated using RGSRMFGEN. Unfortunately, due to the low signal-to-noise the RGS data were not useful.

The OM was operated in imaging mode for the entire observation. Fourteen images were taken in three filters: 5 in $U V W 1$ $(245-320 \mathrm{~nm}), 9$ in $U V M 2(205-245 \mathrm{~nm})$, and 1 in $U V W 2$ (180-225 nm). The average exposure time (800 s) was short for such a faint source and magnitudes could not be calculated for all $U V M 2$ images. The average apparent magnitude in each filter was $U V W 1=16.40 \pm 0.07, U V M 2=16.51 \pm 0.08$, and $U V W 2=16.14 \pm 0.07$, somewhat larger than the ground-based measured $U(309-373 \mathrm{~nm})$ magnitude $m_{U}=15.1 \pm 0.2$ for the whole galaxy (Surace \& Sanders 2000), but not inconsistent when accounting for the different aperture sizes used.

\subsection{Chandra}

Chandra observed Mrk 273 on April 2000 with the Advanced CCD Imaging Spectrometer (ACIS-S3 Back Illuminated chip) for about $47 \mathrm{ks}$. The data relevant to this observation were already published in Paper I, where they were processed following the Standard Data Processing available at that time (R4CU5UPD13.2, January 2001).

Here, data are reprocessed using much more recent versions of the Chandra Interactive Analysis of Observations software (CIAO 3.2) and Chandra Calibration Database (CALDB 3.0.0). The most effective improvements introduced concern the more accurate background subtraction and more careful computation of the effective areas and response matrices.

We start processing data from the level $=1$ event file. We apply the recently released, time-dependent gain correction ${ }^{1}$, which is necessary to adjust the "effective gains", which have been drifting with time due to increasing charge transfer inefficiency. Since the observation was taken in the VFAINT mode, we run the tool acis_process_events to flag probable background events using all the information of the pulse heights in a $5 \times 5$ event island to help distinguishing between good X-ray events and bad events that are most likely associated with cosmic rays. With this procedure, the ACIS particle background can be reduced significantly compared to the standard grade selection ${ }^{2}$. Real X-ray photons are practically not affected by such cleaning (only about $2 \%$ of them are rejected, independently of the energy band, provided there is no pileup).

The data are filtered to include only the standard event grades $0,2,3,4$ and 6 . We finally filter time intervals with high background by performing a $3 \sigma$ clipping of the background level using the script analyze_ltcrv ${ }^{3}$. This yield an effective exposure time of about $43 \mathrm{ks}$ for the ACIS-S3 chip in the energy range $0.3-10 \mathrm{keV}$.

As in Paper I, we separate the spectral analysis of the nuclear emission from the extended soft X-ray halo. The spectrum of the nuclear region of the source is extracted from a circular region of radius $10^{\prime \prime}$ centered at the peak of the hard X-ray

\footnotetext{
1 http://asc.harvard.edu/ciao/threads/acistimegain/

2 http://asc.harvard.edu/cal/Links/Acis/acis/

Cal_prods/vfbkgrnd/

3 http://cxc.harvard.edu /ciao/threads/filter_ltcrv/
} 


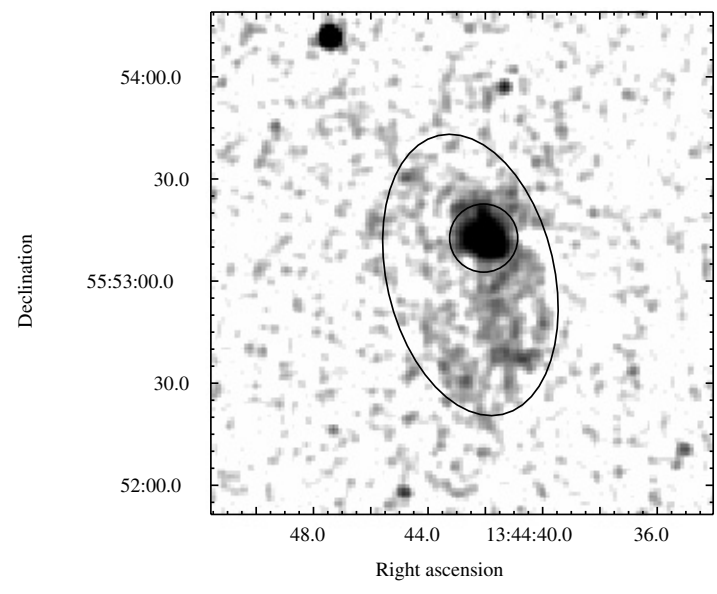

Fig. 1. Adaptively smoothed $0.3-10 \mathrm{keV}$ Chandra image of Mrk 273 with a smoothing scale of 2 pixels $\left(\approx 1^{\prime \prime}\right)$. The circle has a radius of $10^{\prime \prime}$ and it is centered at the peak of the hard X-ray emission. The ellipse has a size of $42^{\prime \prime} \times 25^{\prime \prime}$ and a position angle of $13^{\circ}$. The bright source visible in the upper left corner is Mrk 273x.

Table 1. XMM-Newton and Chandra net number of counts (background subtracted) collected by each instrument in the specified extraction region and spectral range.

\begin{tabular}{llll}
\hline \hline Instrument & $\begin{array}{l}\text { Extraction } \\
\text { region }\end{array}$ & $\begin{array}{l}\text { Energy } \\
{[\mathrm{keV}]}\end{array}$ & $\begin{array}{l}\text { Net number } \\
\text { of counts }\end{array}$ \\
\hline XMM-Newton & & & \\
PN & $50^{\prime \prime}$ & $0.3-10$ & 1680 \\
MOS 1 & $50^{\prime \prime}$ & $0.3-10$ & 590 \\
MOS 2 & $50^{\prime \prime}$ & $0.3-10$ & 520 \\
\hline Chandra & & & \\
ACIS-S & $10^{\prime \prime}$ & $0.3-10$ & 1990 \\
ACIS-S & ellipse & $0.3-2$ & 530 \\
\hline
\end{tabular}

emission, while, for the extended soft halo, we choose the region lying between the inner circle and the ellipse shown in Fig. 1 in order to have the best possible signal-to-noise ratio.

We use the events included in each of the extraction regions defined above to produce a spectrum (PHA) file, which we grouped with a minimum of 20 counts per bin, unless otherwise specified. The background is obtained from a source free circular region of radius $50^{\prime \prime}$ located on the same chip. The background file is then scaled to the source file by the ratio of the geometrical area. The response matrices and the ancillary response matrices of each spectrum are computed with acisspec for the same regions from which the spectra are extracted.

The X-ray spectral analysis has been performed using XSPEC version 11.3.1.

The reported errors on model parameters are at the $90 \%$ confidence level for one interesting parameter $\left(\Delta \chi^{2}=2.71\right)$.

\section{Spectral analysis}

In Table 1 we give a comparison between the XMM-Newton and Chandra data sets. The Chandra ACIS-S spectrum of the nuclear region (inner 10") of Mrk 273 is dominated by the background above $8 \mathrm{keV}$, while the spectrum of the extended soft X-ray halo is source dominated only between 0.5 and $0.9 \mathrm{keV}$. Both the EPIC-PN and the two MOS spectra are source dominated between 0.3 and $10 \mathrm{keV}$, therefore the entire energy range can be analyzed with relatively good signalto-noise. XMM-Newton therefore provides some further constraints on the shape of the continuum between 8 and $10 \mathrm{keV}$.

The extracted PN and ACIS-S light curves do not show any significant (within 30\%) flux or spectral variability.

Throughout our spectral analysis we fix the abundances of the elements to the solar values (Anders \& Grevesse 1989) and we model Galactic absorption with the photoelectric absorption model PHABS in XSPEC, where we fixed the absorbing column density to $N_{\mathrm{g}}=1.09 \times 10^{20} \mathrm{~cm}^{-2}\left(\right.$ HEASARC W3 $\left.\mathrm{nH}^{4}\right)$. Line energies are given in the rest frame of the source.

\subsection{The complex Fe Ka line}

In order to study the properties of the $\mathrm{Fe} \mathrm{K} \alpha$ line we concentrate our spectral analysis only on the hard X-ray (4-10 keV) spectrum of the EPIC-PN. In this energy range the continuum can not be reproduced by a simple unabsorbed power law, since this model gives a negative spectral index and a poor fit. Allowing for extra absorption at the redshift of the source (ZPHABS in XSPEC), we find a much better fit result $\left(\chi_{r}^{2} \simeq 1.2\right)$, but, given the limited statistics available, if both $\Gamma$ and $N_{\mathrm{H}}$ are left free to vary, we find very large uncertainties (e.g. $\Gamma=2.4_{-0.7}^{+1.2}$ ). Fixing the spectral index to the "canonical" value $\Gamma=1.9$ allows us to measure a column density $N_{\mathrm{H}}=(6.2 \pm 1.5) \times 10^{23} \mathrm{~cm}^{-2}$ intrinsic to the source. As displayed in Fig. 2, the fit is still not completely satisfactory $\left(\chi^{2} /\right.$ d.o.f $\left.=24.5 / 20\right)$, mainly because of the presence of a feature in the spectrum at about $6.4 \mathrm{keV}$, which can be identified with an $\mathrm{Fe} \mathrm{K} \alpha$ emission line. A significant improvement in the fit is obtained when a single broad Gaussian is added to the model $\left(\chi^{2} /\right.$ d.o.f. $\left.=15.2 / 17\right)$. The line centroid is at $6.51 \pm 0.19 \mathrm{keV}$ and the line is found to be broad $\left(\sigma=0.26_{-0.17}^{+0.37} \mathrm{keV}\right)$, with an equivalent width $E W=560_{-330}^{+490} \mathrm{eV}$.

Such a broad iron line $\left(F W H M \sim 29000 \mathrm{~km} \mathrm{~s}^{-1}\right)$ could, in principle, originate from the accretion disc, since the primary radiation is obscured only up to about $4-5 \mathrm{keV}$ and therefore should only partly affect the photons at the iron line energy. We try to model the iron line profile with the DISKLINE model in XSPEC for a Schwarzschild black hole, which parameterizes the radial emissivity of the disc as a power law (i.e. $\propto r^{-q}$ ). We fix the line rest energy to $6.4 \mathrm{keV}$, the emissivity of the disc $q$ to 2 , the inner and outer radii respectively to 6 and to $1000 r_{\mathrm{g}}$ and the inclination angle to $30^{\circ}$. We find an equivalent width $E W=$ $520 \pm 300 \mathrm{eV}$ and a fit result statistically equivalent to the one obtained with a single broad Gaussian $\left(\chi^{2} /\right.$ d.o.f $\left.=17.1 / 19\right)$. Unfortunately, the statistics are too poor to derive any physical properties of the disc.

The hypothesis of a broad iron line originating from the disc cannot be ruled out, but the high column density, which is obscuring the AGN up to about $4 \mathrm{keV}$, certainly makes the

\footnotetext{
4 http://heasarc.gsfc.nasa.gov/cgi-bin/Tools/w3nh/ w3nh.pl
} 


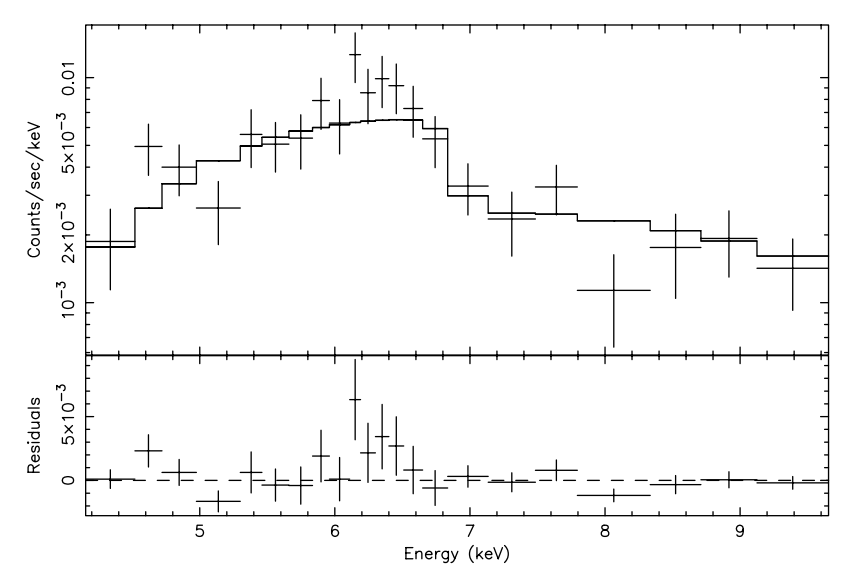

Fig. 2. The 4.0-10 keV EPIC-PN spectrum fitted with a simple absorbed power law model ( $\Gamma$ is fixed to 1.9 ). Residuals are found at $\mathrm{Fe} \mathrm{K} \alpha$ emission line energy (at about $6.5 \mathrm{keV}$ ).

detection of the redshifted wing of a relativistic iron line profile much less feasible. A more likely explanation for the prominent iron line feature observed may be given in terms of a complex profile due to the superposition of, at least, two unresolved line components: a neutral iron line at $6.4 \mathrm{keV}$, probably originating from a Compton-thick torus, and an ionized (Fe XXV) iron line at $6.7 \mathrm{keV}$. Indeed, the latter could be produced either by some diffuse collisionally ionized plasma at temperature $k T \geq 5 \mathrm{keV}$, as observed in at least another two ULIRGs (namely NGC 6240, Boller et al. 2003; Netzer et al. 2005 and Arp 220, Iwasawa et al. 2005), or by reflection off some photoionized gas surrounding the nucleus, as often seen in Compton-thick Seyfert galaxies (e.g. Circinus, Sambruna et al. 2001; NGC 1068, Kinkhabwala et al. 2002; NGC 4945, Done et al. 2003; Mrk 3, Bianchi et al. 2005).

When the line profile is modeled with two narrow $(\sigma=$ $0 \mathrm{eV}$ ) Gaussian lines with energies fixed at 6.4 and $6.7 \mathrm{keV}$ the result is statistically equivalent to a single broad line model $\left(\chi^{2} /\right.$ d.o.f. $\left.=15.5 / 18\right)$. In this case we measure an equivalent width $E W=165_{-110}^{+120} \mathrm{eV}$ and $E W=120 \pm 95 \mathrm{eV}$ for the neutral and for the ionized component respectively. Finally, we find no improvement in the fit for the addition of a third ionized iron line at $6.97 \mathrm{keV}$ from Fe XXVI. However upper limits on the flux $\left(<0.18 \times 10^{-13} \mathrm{erg} \mathrm{cm}^{-2} \mathrm{~s}^{-1}\right)$ and the equivalent width $(<85 \mathrm{eV})$ of this line can be derived.

In the following we discuss two distinct models capable of reproducing the broad band spectrum as well as the possible ionized iron lines: one through thermal emission, therefore involving the presence of a hot gas associated with a starburst, and the other one through reflection of the primary continuum by some optically thin photoionized gas.

\subsection{The thermal emission model}

At first we concentrate our analysis on the EPIC-PN spectrum. The better statistics, especially at high energies, permit more robust constraints of the model parameters. Once the best fit for the PN is found, we perform a combined fit of the PN and the two MOS, applying the same model, in order to further improve the statistics.

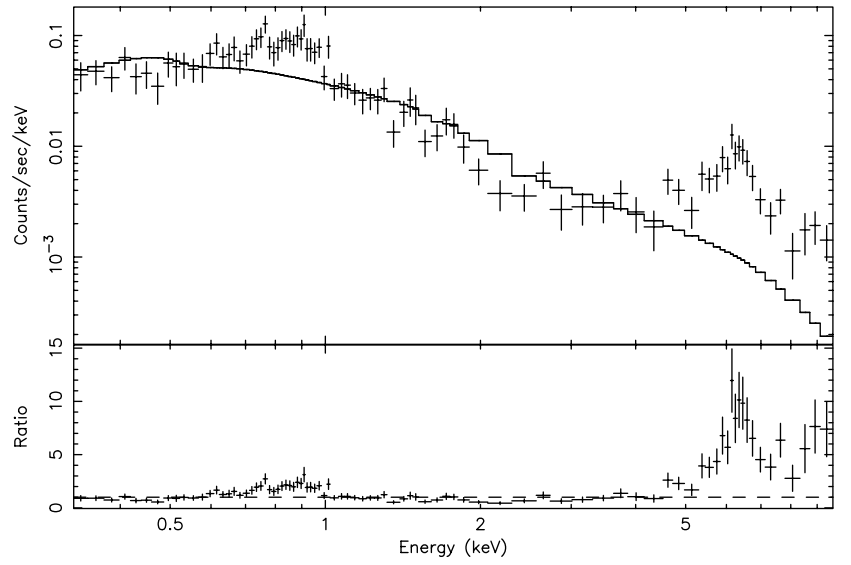

Fig. 3. A simple absorbed power law model applied to the EPICPN spectrum of Mrk 273. Strong residuals (data/model) are clearly present above $4 \mathrm{keV}$ and also between 0.6 and $1 \mathrm{keV}$.

When the 0.3-10 keV EPIC-PN spectrum is modeled with a simple absorbed (Galactic plus intrinsic) power law, we obtain a statistically unacceptable fit $\left(\chi_{r}^{2}>4\right)$. Strong residuals remain primarily above $4 \mathrm{keV}$, but also between 0.6 and $1 \mathrm{keV}$ (Fig. 3).

As a starting point for our spectral modelling we adopt the best fit absorbed power law plus Gaussian model for the 4-10 keV band (see Sect. 3.1) and extrapolate to $0.3 \mathrm{keV}$. A very prominent soft excess is clearly noticeable. Since we intend to investigate both the origin of the soft excess and of the possible ionized iron, in the following we fix the parameters of the Gaussian profile to $E=6.4 \mathrm{keV}$ and $\sigma=0 \mathrm{eV}$. This will be considered as our baseline model.

Diffuse, optically thin, thermal plasma emission is often observed in Starburst galaxies and ULIRGs. It is typically associated with star-forming regions and shock-heated gas in merging systems. We try to model the soft, extended, X-ray emission with a thermal spectrum from a collisionally ionized plasma, using the APEC model (Smith et al. 2001) in XSPEC. A single temperature plasma component gives a considerable improvement to the fit with respect to the baseline model $\left(\chi^{2} /\right.$ d.o.f. $=$ $259 / 82$ ), but can not simultaneously account for the observed peak at about $0.7-0.9 \mathrm{keV}$ and the possible ionized iron lines.

Therefore, we add to the baseline model two collisionally ionized plasma components of different temperatures. The free parameters are now the temperature and the normalization of each plasma component, the column density of the absorber at the redshift of the source, the normalization of the power law and the normalization of the Gaussian line. The best fit parameters for this model are given in Table 2. A plasma temperature of $\sim 0.7 \mathrm{keV}$ is needed in order to model the peak in the emission at low energies, while a much hotter $(\sim 5 \mathrm{keV})$ plasma is required to fit part of the high energy continuum and also to give rise to the ionized iron line at $6.7 \mathrm{keV}$. The second, hotter, plasma is required with a high statistical significance ( $\Delta \chi^{2} \simeq 175$ for the addition of two free parameters). However the fit is still not completely satisfactory $\left(\chi^{2} /\right.$ d.o.f. $\left.=84.2 / 80\right)$ due to the presence of some excess below about $0.6 \mathrm{keV}$. 
Table 2. XMM-Newton EPIC-PN spectral fitting results when the thermal emission model is applied (2 APEC: two temperature model; 3 APEC: three temperature model). The last column displays the result of the combined PN+MOS fit with the 3 APEC model. Fluxes are given in units of $10^{-13} \mathrm{erg} \mathrm{cm}^{-2} \mathrm{~s}^{-1}$, luminosities in units of $10^{43} \mathrm{erg} \mathrm{s}^{-1}$; both are corrected for absorption. ${ }^{*}$ denotes a fixed parameter.

\begin{tabular}{llll}
\hline \hline & 2 APEC & 3 APEC & 3 APEC \\
& PN & PN & PN + MOS \\
\hline Apec(1) & & & \\
$k T[\mathrm{keV}]$ & $4.7_{-1.1}^{+2.2}$ & $6.1_{-2.0}^{+4.3}$ & $5.4_{-1.3}^{+2.6}$ \\
$f_{0.3-10 \mathrm{keV}}$ & $1.40 \pm 0.15$ & $1.36^{-1} \pm .18$ & $1.43 \pm 0.14$ \\
\hline Apec(2) & & & \\
$k T[\mathrm{keV}]$ & $0.66_{-0.04}^{+0.06}$ & $0.75 \pm 0.07$ & $0.78 \pm 0.08$ \\
$f_{0.3-10 \mathrm{keV}}$ & $0.63_{-0.07}^{+0.11}$ & $0.55_{-0.15}^{+0.12}$ & $0.47_{-0.15}^{+0.13}$ \\
\hline Apec(3) & & & \\
$k T[\mathrm{keV}]$ & - & $0.26_{-0.06}^{+0.08}$ & $0.31_{-0.07}^{+0.09}$ \\
$f_{0.3-10 \mathrm{keV}}$ & - & $0.23_{-0.10}^{+0.14}$ & $0.23_{-0.09}^{+0.13}$ \\
\hline Power-law & & & \\
$N_{\mathrm{H}}\left[10^{22} \mathrm{~cm}{ }^{-2}\right]$ & $68_{-15}^{+19}$ & $69_{-19}^{+16}$ & $69_{-14}^{+15}$ \\
$\Gamma$ & $1.9^{*}$ & $1.9^{*}$ & $1.9^{*}$ \\
$f_{0.3-10 \mathrm{keV}}$ & $44_{-13}^{+21}$ & $44_{-13}^{+27}$ & $46_{-12}^{+17}$ \\
\hline Fe K $\alpha$ & & & \\
$E W[\mathrm{eV}]$ & $210_{-140}^{+190}$ & $213_{-143}^{+198}$ & $130 \pm 120$ \\
$f_{0.3-10 \mathrm{keV}}$ & $0.48_{-0.34}^{+0.43}$ & $0.49_{-0.33}^{+0.46}$ & $0.31 \pm 0.29$ \\
\hline Total & & & \\
$f_{0.3-10 \mathrm{keV}}$ & 46.6 & 46.6 & 48.7 \\
$L_{0.3-10 \mathrm{keV}}$ & 1.54 & 1.54 & 1.66 \\
\hline$\chi^{2} / \mathrm{d} . \mathrm{o.f}$. & $84.2 / 80$ & $63.8 / 78$ & $130.0 / 134$ \\
\hline
\end{tabular}

The addition of a third, lower temperature, plasma component provides a further improvement to the fit $\left(\Delta \chi^{2} \simeq 20\right.$ for the addition of two free parameters). In this case we find an undoubtedly acceptable result $\left(\chi^{2} /\right.$ d.o.f. $\left.=63.8 / 78\right)$. The lowest temperature component is required with a probability of more than $99.99 \%$ according to an F-test. Its temperature $(k T=$ $0.26_{-0.06}^{+0.08} \mathrm{keV}$ ) is consistent with the one derived from the spectrum of the extended hot gas halo $(k T=0.38 \pm 0.04 \mathrm{keV})$ observed in the Chandra image (see Sect. 4.2).

The best fit to the $0.3-10 \mathrm{keV}$ PN spectrum is therefore obtained using three thermal plasma components with different temperatures, a highly absorbed power law and a neutral $\mathrm{Fe} \mathrm{K} \alpha$ line (Fig. 4). According to this model the emission below about $4 \mathrm{keV}$ would be purely thermal. We will discuss the possible implications of these new findings in Sect. 5.

Simultaneously fitting the PN and the two MOS data results in an equivalent fit, as shown in Fig. 4. All the parameters are consistent with those obtained using the PN only (see Table 2). Combining the PN and the MOS, we find a temperature $k T=$ $0.31_{-0.07}^{+0.09} \mathrm{keV}$ for the coolest thermal component, which is in very good agreement with the one measured by Chandra for the hot extended halo.

There is no need for further absorption at the redshift of the source for any of the three thermal components. When left free to vary the absorbing column density of each component is consistent with the Galactic value and does not improve the fit $\left(\Delta \chi^{2}<1\right.$ for the addition of one free parameter).
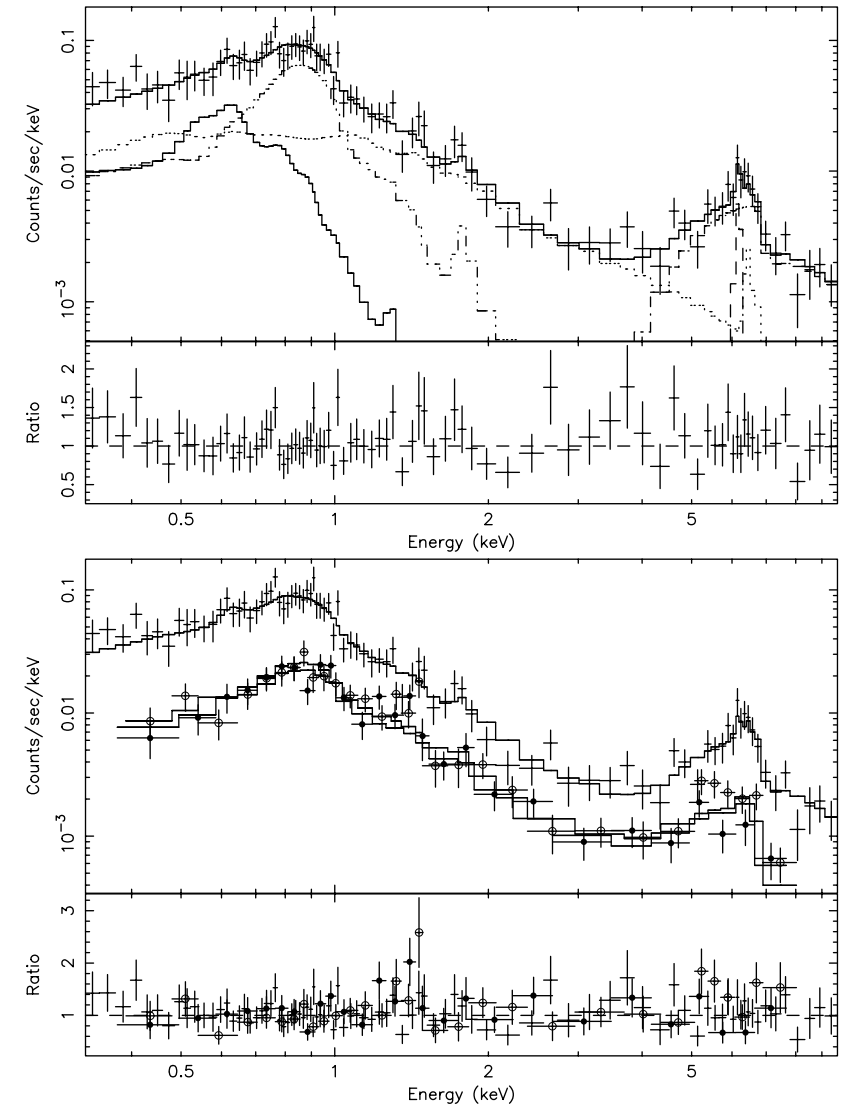

Fig. 4. (Upper panel) The EPIC-PN spectrum of Mrk 273 fitted with three different temperature APEC models, a highly absorbed power law and a neutral $\mathrm{Fe} \mathrm{K} \alpha$ line. Each model component is displayed. (Lower panel) The EPIC-PN and the two MOS combined fit with the same model. The closed and open circles correspond to the two MOS data sets. The best fit parameters are given in Table 2 .

\subsection{The photoionized gas model}

An alternative interpretation for the origin of the soft X-ray continuum and the ionized iron line observed in the XMMNewton spectrum is scattering of the primary radiation by some photoionized, Compton-thin, hot gas surrounding the AGN. Ionized $\mathrm{Fe} \mathrm{K} \alpha$ emission lines from $\mathrm{H}$ - and $\mathrm{He}$-like iron ions are expected to be produced through recombination and resonant scattering (Bianchi \& Matt 2002).

The analysis of the Chandra image in Paper I revealed that the emission below $1 \mathrm{keV}$ is extended over a region of radius $\sim 7.5 \mathrm{kpc}$ surrounding the nucleus and, partly, over a much larger region $(\sim 45 \mathrm{kpc})$ encompassing the tidal tail. A pure reflection model cannot account for such extended $\mathrm{X}$-ray emission, which must be of thermal origin. If we model the $0.3-10 \mathrm{keV}$ PN continuum with a highly absorbed (primary) plus a less absorbed (reflected) power law having the same spectral index, we find an unacceptable fit $\left(\chi_{r}^{2} \simeq 2.6\right)$. Therefore we added to the model two narrow Gaussian lines, at $6.4 \mathrm{keV}$ from neutral iron and at $6.7 \mathrm{keV}$ from Fe XXV, plus a hot collisionally ionized plasma component. In this case the fit result is acceptable $\left(\chi^{2} /\right.$ d.o.f $\left.=66.9 / 79\right)$. However, there is no need for an additional lower temperature thermal plasma component, which is clearly detected by Chandra. 
Table 3. XMM-Newton EPIC-PN spectral fitting results using the photoionized gas model described in the text. Fluxes are given in units of $10^{-13} \mathrm{erg} \mathrm{cm}^{-2} \mathrm{~s}^{-1}$, luminosities in units of $10^{43} \mathrm{erg} \mathrm{s}^{-1}$; both are corrected for absorption. ${ }^{*}$ denotes a fixed parameter.

\begin{tabular}{lll}
\hline \hline & 1 APEC & 2 APEC \\
\hline Apec(1) & & \\
$k T[\mathrm{keV}]$ & $0.66_{-0.04}^{+0.07}$ & $0.76_{-0.07}^{+0.08}$ \\
$f_{0.3-10 \mathrm{keV}}$ & $0.62 \pm 0.07$ & $0.52 \pm 0.11$ \\
\hline Apec(2) & & \\
$k T[\mathrm{keV}]$ & - & $0.26_{-0.03}^{+0.08}$ \\
$f_{0.3-10 \mathrm{keV}}$ & - & $0.23_{-0.10}^{+0.11}$ \\
\hline Power-law(1) & & \\
$N_{\mathrm{H}}\left[10^{22} \mathrm{~cm}^{-2}\right]$ & $68_{-15}^{+17}$ & $65_{-12}^{+15}$ \\
$\Gamma$ & $1.9^{*}$ & $1.9^{*}$ \\
$f_{0.3-10 \mathrm{keV}}$ & $41_{-12}^{+21}$ & $39_{-11}^{+20}$ \\
\hline Power-law(2) & & \\
$N \mathrm{H}\left[10^{20} \mathrm{~cm}{ }^{-2}\right]$ & $7^{*}$ & $7^{*}$ \\
$\Gamma$ & $1.9^{*}$ & $1.9^{*}$ \\
$f_{0.3-10 \mathrm{keV}}$ & $1.7 \pm 0.2$ & $1.5 \pm 0.2$ \\
\hline Fe K $\alpha$ & & \\
$E[\mathrm{keV}]$ & $6.4^{*}$ & $6.4^{*}$ \\
$E W[\mathrm{eV}]$ & $186_{-125}^{+165}$ & $185_{-122}^{+159}$ \\
$f_{0.3-10 \mathrm{keV}}$ & $0.50_{-0.34}^{+0.44}$ & $0.47_{-0.31}^{+0.40}$ \\
\hline Fe XXV & & \\
$E[\mathrm{keV}]$ & $6.7^{*}$ & $6.7^{*}$ \\
$E W[\mathrm{eV}]$ & $121_{-102}^{+110}$ & $124_{-103}^{+110}$ \\
$f_{0.3-10 \mathrm{keV}}$ & $0.34_{-0.29}^{+0.31}$ & $0.33_{-0.27}^{+0.29}$ \\
\hline Total & & \\
$f_{0.3-10 \mathrm{keV}}$ & 44.5 & 42.1 \\
$L_{0.3-10 \mathrm{keV}}$ & 1.47 & 1.39 \\
\hline$\chi^{2} / \mathrm{d} . \mathrm{o.f}$. & $84.9 / 80$ & $64.0 / 78$ \\
\hline & &
\end{tabular}

This apparent discrepancy between the two data sets may be reconciled simply by taking into account the absorption of the secondary power law, which is apparently negligible in the XMM-Newton spectrum, but $\sim 7 \times 10^{20} \mathrm{~cm}^{-2}$ in the Chandra spectrum of the "nuclear" region (see Sect. 4.1). Therefore we fix the column density of the material obscuring the reflected power law to the value measured by Chandra. The free parameters of the model are: the temperature and the normalization of the thermal component; the column density of the absorber at the redshift of the source, which is obscuring only the primary power law; the normalizations of the two power law components; and the normalizations of the two Gaussian lines.

When this model is applied to the PN spectrum the fit result is still not completely satisfactory $\left(\chi^{2} /\right.$ d.o.f $\left.=84.9 / 80\right)$, mostly due to some excess still present below $0.6 \mathrm{keV}$. The best fit parameters for this model are given in Table 3.

The addition of a second, lower temperature, plasma component results in a considerable improvement to the fit $\left(\Delta \chi^{2} \simeq\right.$ 20 for the addition of two free parameters, $\chi^{2} /$ d.o.f. $=$ 64.0/78). Figure 5 shows the best fit obtained for this model. The cooler thermal plasma component is required with a probability of more than $99.99 \%$ according to an F-test. The temperature found $k T=0.26_{-0.03}^{+0.08} \mathrm{keV}$ is consistent, within the uncertainties, with that measured by Chandra for the extended hot gas halo.

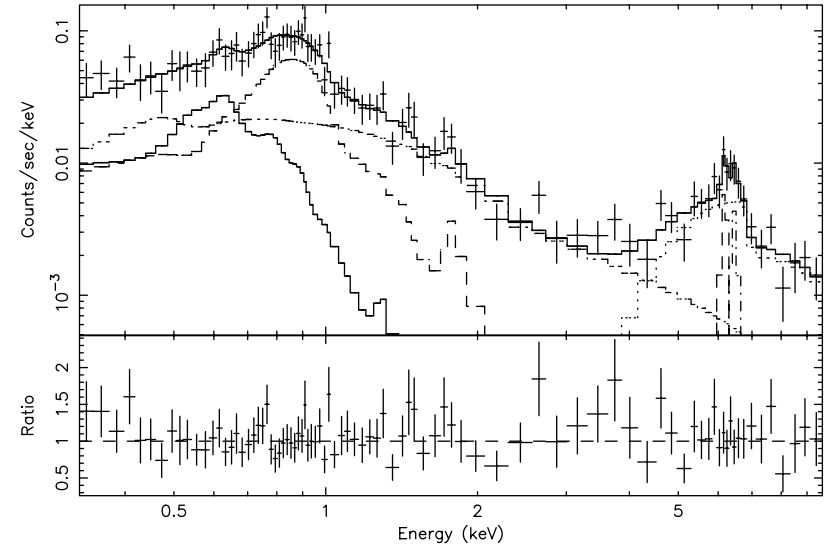

Fig. 5. The EPIC-PN spectrum fitted with two thermal plasma components, a highly absorbed and a less absorbed power law with the same spectral index, plus a narrow $\mathrm{Fe} \mathrm{K} \alpha$ line at $6.4 \mathrm{keV}$. The best fit parameters for this model are given in Table 3.

The flux of the reflected power law amounts to $2-6 \%$ of the primary component. From this ratio we can estimate the column density of the reflecting material. Assuming a covering factor of 0.5 , the column density of the photoionized gas would be approximately $10^{23} \mathrm{~cm}^{-2}$. The equivalent widths of the ionized iron lines expected to be produced by a hot gas with such a density are consistent with the measured values within the uncertainties.

The best fit model obtained here is similar to the one presented in Paper I, with the only noticeable differences being a slightly higher absorbing column density obscuring the primary radiation and a slightly lower temperature of the cooler thermal component.

\section{Comparison with Chandra}

For a detailed spatial analysis of the Chandra X-ray image of Mrk 273 we refer the reader to Paper I. Here, as in Paper I, we extract separately the spectra of the nuclear region and that of the extendend soft halo from the two regions shown in Fig. 1, in order to have the best possible signal-to-noise ratio.

\subsection{The inner $10^{\prime \prime}$ region}

We apply to the Chandra spectrum of the inner region $\left(10^{\prime \prime} \sim\right.$ $7.5 \mathrm{kpc}$ surrounding the AGN) both models successfully tested in Sect. 3 for the XMM-Newton data.

The thermal emission model, which was not tested in Paper I, when applied to the Chandra spectrum of the "nuclear" region, also gives a good fit $\left(\chi^{2} /\right.$ d.o.f $\left.=65.0 / 75\right)$. The best fit parameters are given in Table 4 . In this case, only two collisionally ionized plasma components are required, as shown in Fig. 6.

The photoionized gas model, which we discuss here, is similar to the one presented in Paper I. It consists of a highly absorbed (primary) plus a less absorbed (secondary) power law having the same spectral index, two $\mathrm{Fe} \mathrm{K} \alpha$ lines at 6.4 and $6.7 \mathrm{keV}$ and a single thermal plasma component. This model 
Table 4. Chandra ACIS-S spectral fitting results when the thermal emission model (Model 1) and the photoionized gas model (Model 2) are applied to the spectrum of the inner $10^{\prime \prime}$. The last column refers to the spectrum of the extended hot gas halo instead. Fluxes are given in units of $10^{-13} \mathrm{erg} \mathrm{cm}^{-2} \mathrm{~s}^{-1}$, luminosities in units of $10^{43} \mathrm{erg} \mathrm{s}^{-1}$; both are corrected for absorption. ${ }^{*}$ denotes a fixed parameter.

\begin{tabular}{llll}
\hline \hline & $\begin{array}{l}\text { Model 1 } \\
\text { Inner 10" }\end{array}$ & $\begin{array}{l}\text { Model 2 } \\
\text { Inner 10" }\end{array}$ & $\begin{array}{l}\text { Hot Gas } \\
\text { Halo }\end{array}$ \\
\hline Apec(1) & & & \\
$k T[\mathrm{keV}]$ & $7.8_{-2.8}^{+7.5}$ & - & - \\
$f_{0.3-10 \mathrm{keV}}$ & $1.60_{-0.17}^{+0.20}$ & - & - \\
\hline Apec(2) & & & \\
$k T[\mathrm{keV}]$ & $0.80_{-0.05}^{+0.04}$ & $0.81 \pm 0.05$ & - \\
$f_{0.3-10 \mathrm{keV}}$ & $0.37 \pm 0.06$ & $0.33_{-0.05}^{+0.03}$ & - \\
\hline Apec(3) & & & \\
$k T[\mathrm{keV}]$ & - & - & $0.38 \pm 0.04$ \\
$f_{0.3-10 \mathrm{keV}}$ & - & - & $0.30 \pm 0.04$ \\
\hline Power-law(1) & & & \\
$N \mathrm{H}\left[10^{22} \mathrm{~cm}{ }^{-2}\right]$ & $41 \pm 6$ & $39 \pm 5$ & - \\
$\Gamma$ & $1.9^{*}$ & $1.9^{*}$ & - \\
$f_{0.3-10 \mathrm{keV}}$ & $53_{-9}^{+12}$ & $53_{-9}^{+10}$ & - \\
\hline Power-law(2) & & & \\
$N \mathrm{H}\left[10^{20} \mathrm{~cm}{ }^{-2}\right]$ & - & $6.8_{-2.0}^{+2.3}$ & - \\
$\Gamma$ & - & $1.9^{*}$ & - \\
$f_{0.3-10 \mathrm{keV}}$ & - & $1.7 \pm 0.2$ & - \\
\hline Fe K $\alpha$ & & & \\
$E$ & $6.34 \pm 0.04$ & $6.34 \pm 0.04$ & - \\
$E W[\mathrm{eV}]$ & $255 \pm 125$ & $242 \pm 120$ & - \\
$f_{0.3-10 \mathrm{keV}}$ & $0.72 \pm 0.35$ & $0.69 \pm 0.34$ & - \\
\hline Total & & & \\
$f_{0.3-10 \mathrm{keV}}$ & 55.4 & 55.9 & 0.3 \\
$L_{0.3-10 \mathrm{keV}}$ & 1.84 & 1.86 & 0.01 \\
\hline$\chi^{2} / \mathrm{d} . \mathrm{o.f}$. & $65.0 / 75$ & $62.0 / 75$ & - \\
\hline & & & \\
\hline
\end{tabular}

also provides a good fit $\left(\chi^{2} /\right.$ d.o.f $=62.0 / 75$, see Fig. 7$)$. A column density of $N_{\mathrm{H}}=6.8_{-2.8}^{+2.3} \times 10^{20} \mathrm{~cm}^{-2}$ obscuring the secondary power law is needed, in agreement with Paper I and the $\mathrm{XMM}-$ Newton results.

A narrow, unresolved, $\mathrm{Fe} \mathrm{K} \alpha$ line is detected at about $3 \sigma$ significance in the ACIS-S spectrum. Its centroid is found at $6.34 \pm 0.04 \mathrm{keV}$, which is slightly lower than the expected value of $6.4 \mathrm{keV}$ from neutral iron, probably due to uncertainties in the calibration. No ionized iron lines are required by the data; however, we derive upper limits on the fluxes of the Fe XXV and Fe XXVI lines (respectively $<0.37 \times 10^{-13}$ and $<0.39 \times$ $10^{-13} \mathrm{erg} \mathrm{cm}^{-2} \mathrm{~s}^{-1}$ ), which are consistent with those measured by XMM-Newton.

The best fit parameters for both models (summarized in Table 4) are consistent, within the uncertainties, with those found by XMM-Newton. The only exception is in the value of the column density obscuring the primary radiation, which is slightly different between the two observations $(41 \pm 6$ and $69_{-19}^{+16} \times 10^{22} \mathrm{~cm}^{-2}$ for the Chandra and the XMM-Newton observation respectively).

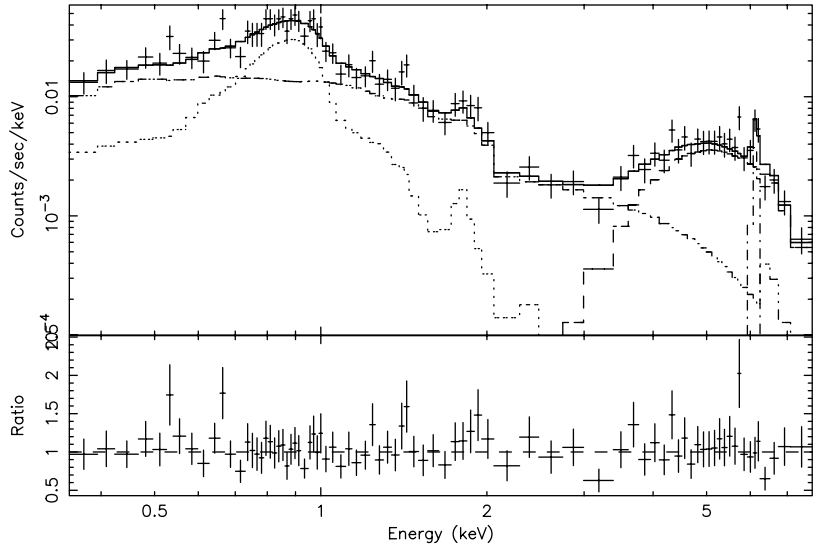

Fig. 6. The 0.3-8 keV ACIS-S spectrum of the inner 10" region fitted with two thermal plasma components, a highly absorbed power law and a narrow $\mathrm{Fe} \mathrm{K} \alpha$ line at $6.4 \mathrm{keV}$. The best fit parameters are given in Table 4.

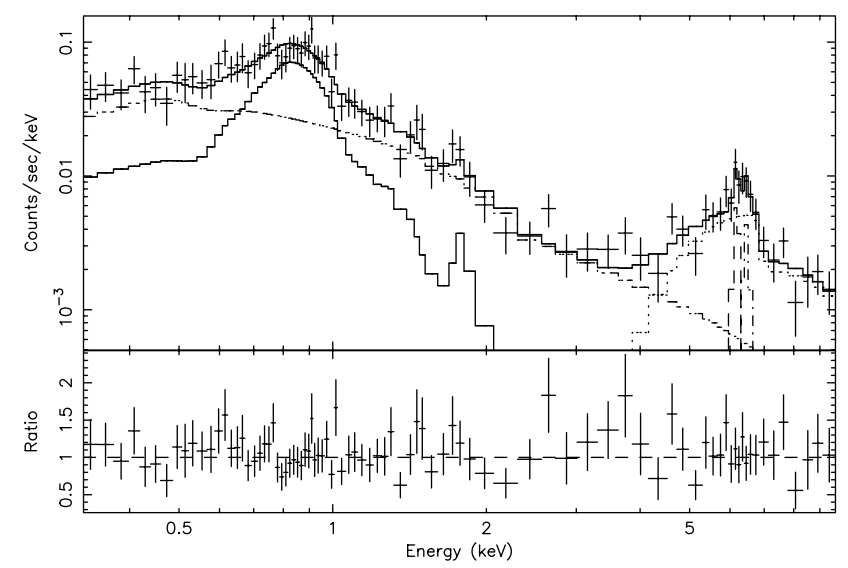

Fig. 7. The $0.3-8 \mathrm{keV}$ ACIS-S spectrum of the inner $10^{\prime \prime}$ region fitted with a single thermal plasma component, a highly absorbed and a less absorbed power law with the same spectral index, plus a narrow $\mathrm{Fe}$ $\mathrm{K} \alpha$ line at $6.4 \mathrm{keV}$. The best fit parameters are given in Table 4 .

\subsection{The hot gas halo}

The ACIS-S spectrum of the extended hot gas halo, which is extracted from the region defined in Sect. 2.2, is source dominated only in the $0.5-0.9 \mathrm{keV}$ band. Given the limited amount of photons (315) detected in this band, we prefer to use Cash statistics on the unbinned spectrum. As displayed in Fig. 8, the spectrum is consistent with a single temperature plasma component with $k T=0.38 \pm 0.04 \mathrm{keV}$. We performed a Monte Carlo calculation to test the goodness of the fit and we found that $63 \%$ of the $10^{4}$ simulated spectra have a fit statistics less than that for the data. A fraction of approximately $50 \%$ is expected, if the observed spectrum was produced by the model. If the $\chi^{2}$ statistics is applied to the spectrum grouped to have a minimum of 20 counts per bin, fixing the model parameters to those previously found (see Table 4), we obtain $\chi^{2} /$ d.o.f $=15 / 11$.

In this analysis we adopted a more conservative approach in subtracting the background in comparison to Paper I. This resulted in a slightly lower temperature for the extended hot gas halo with respect to the previous analysis, but in very good agreement with the XMM-Newton results. 


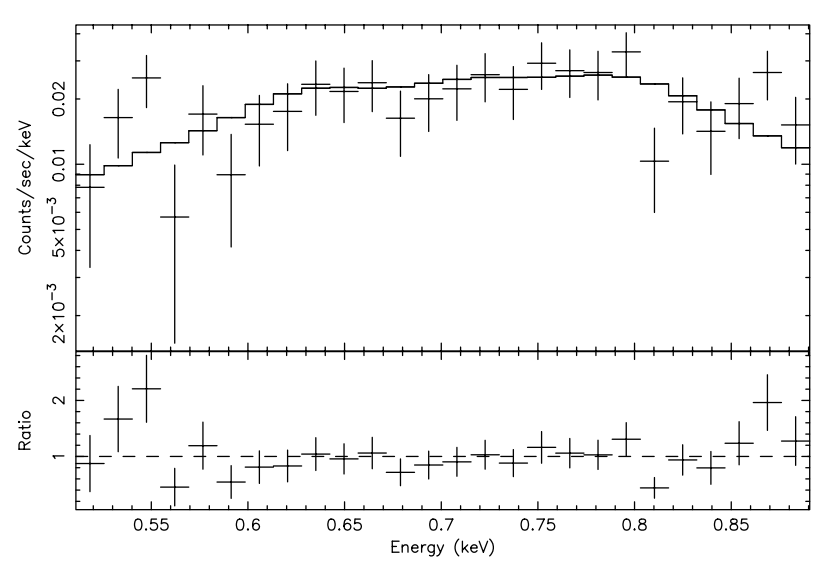

Fig. 8. The 0.5-0.9 keV unbinned spectrum of the extended hot gas halo. A single APEC model with a temperature of $0.38 \pm 0.04 \mathrm{keV}$ provides a satisfactory fit.

\section{Discussion}

A broad $\mathrm{Fe} \mathrm{K} \alpha$ emission line with $\sigma=0.26_{-0.17}^{+0.37} \mathrm{keV}$ is detected at high significance in the broad band $(0.3-10 \mathrm{keV})$ EPIC-PN spectrum $\left(\Delta \chi^{2}=11.4\right.$ for three additional free parameters, corresponding to more than $99 \%$ confidence level). The hypothesis of a broad iron line originating from the disc is plausible, but the high column density, obscuring the AGN up to about $4 \mathrm{keV}$, makes the detection of the redshifted wing of a relativistic iron line profile more difficult. The most likely explanation for such a broad line profile is the superposition of a narrow, unresolved, neutral iron line at $6.4 \mathrm{keV}$, probably associated with the AGN torus, and a blend of Fe XXV and Fe XXVI lines, associated with a hot plasma, in which this transitions are either thermally excited or induced by photoionization. Unfortunately, the modest statistics do not allow us to individually resolve the three possible lines. However the fluxes and equivalent widths measured by Chandra and XMMNewton are consistent with each other.

There is no evidence for extra absorbing material obscuring any of the thermal plasma components. This could be due to a non-spherical geometry of the gas surrounding the nuclear region, given the complex morphology of this merger, or it could be simply due to the low statistics. A slightly different column density is found to be obscuring the primary radiation between the two observations. Again this could be, associated with the complex morphology of the merger, since, for instance, the projected separation between the two nuclei is $\sim 1^{\prime \prime}$, or it could be an effect due to the low statistics available at high energies.

According to the thermal emission model, the temperatures of the three plasma components found in Mrk 273 are remarkably similar to those observed in other ULIRGs (e.g. NGC 6240 and Arp 220) and in the local starburst galaxy NGC 253 (Pietsch et al. 2001). The thermal emission at $\sim 0.3 \mathrm{keV}$, as clearly shown from the Chandra image, extends on a large scale $(\sim 45 \mathrm{kpc})$ and embraces the long tidal tail of the merger. Therefore, it must be associated with hot gas distributed in the halo. Interestingly, thermal emission at $\sim 0.7 \mathrm{keV}$ seems to be ubiquitous in the spectra of ULIRGs, probably being associated with a nuclear or circumnuclear starburst (Franceschini et al. 2003). The presence of a high temperature $(\gtrsim 5 \mathrm{keV})$ thermal component is less frequently observed, but not so unusual, being also detected in the XMM-Newton spectra of NGC 6240 (Boller et al. 2003) and Arp 220 (Iwasawa et al. 2005). The ratio of the $2-10 \mathrm{keV}$ unabsorbed X-ray luminosity of the highest temperature thermal component to the bolometric luminosity in Mrk 273 is $L_{2-10 \mathrm{keV}} / L_{\mathrm{FIR}} \simeq 7 \times 10^{-5}\left(L_{\mathrm{FIR}}=L\left(8-1000 \mu \mathrm{m} \sim L_{\mathrm{bol}}\right), \mathrm{a}\right.$ factor of 20 smaller than in NGC 6240 and a factor of 5 larger than in Arp 220.

On the other hand, the second model investigated suggests that a secondary hard X-ray component originates from the reflection of the primary continuum on some photoionized circumnuclear material. This is often observed in Compton-thick Seyfert 2 galaxies (Sambruna et al. 2001; Kinkhabwala et al. 2002; Done et al. 2003; Bianchi et al. 2005), which, in some cases, also show evidence for starburst activity (e.g. Circinus, Ruiz et al. 2001; NGC 1068, Smith \& Wilson 2003). As was observed in Paper I, two bright clumps of soft X-ray emission coincide with [OIII] emission. This evidence seems to support the hypothesis of a photoionized gas surrounding the nucleus, since in several Compton-thick Seyfert galaxies, the extended soft X-ray emission expected to be produced by reflection from that photoionized gas has been observed to coincide with the [OIII] emission and the ionization cone (e.g. Sako et al. 2000; Young et al. 2001).

The absorption-corrected hard X-ray luminosity of Mrk 273 is $L_{2-10 \mathrm{keV}} \sim 7 \times 10^{42} \mathrm{erg} \mathrm{s}^{-1}$, which corresponds to $\sim 2 \times 10^{-3}$ of the far-IR luminosity $\left(L_{\mathrm{FIR}} \sim 10^{12} L_{\odot}\right.$, e.g. Genzel et al. 1998). Such a ratio approaches values of pure starbursts and is orders of magnitude smaller than in Comptonthin AGNs (Risaliti et al. 2000). According to the thermal emission model, the absorption-corrected $0.3-10 \mathrm{keV}$ luminosity due to the three thermal components would be approximately $0.7 \times 10^{42} \mathrm{erg} \mathrm{s}^{-1}$. Comparable soft X-ray luminosities have been measured for NGC 6240 (Netzer et al. 2005) and other ULIRGs in which the starburst contribution to the bolometric luminosity is dominant (Franceschini et al. 2003). From the photoionized gas model the resulting thermal contribution to the X-ray luminosity is about a factor of 3 smaller. For Mrk 273 the unobscured AGN X-ray luminosity is a more modest fraction of the bolometric luminosity than in typical AGN. This suggests additional strong star formation which will naturally explain the thermal X-ray components invoked in one of our two scenarios. The presence of strong star formation agrees with the detection from mid-infrared spectroscopy (Genzel et al. 1998) of both very powerful star formation and an AGN in Mrk 273.

Besides the two models discussed above, other interpretations are possible. X-ray emission from luminous radio supernovae like SN1995J (Fox et al. 2000), or the integrated contribution from low- and high-mass X-ray binaries, as observed from the spectra of local starburst galaxies (Persic \& Rephaeli 2002), may both give rise to a very hot $(k T \sim 5 \mathrm{keV})$ thermal component, or a $\Gamma \sim 2$ power law. Following Kennicutt (1998), the star formation rate estimated assuming that the entire $L_{\mathrm{FIR}}$ is due to starburst would be $\sim 200 M_{\odot} \mathrm{yr}^{-1}$. Therefore, the inferred supernova rate is $\sim 2 \mathrm{yr}^{-1}$, which is in agreement with the value estimated from radio observations Bondi et al. (2005). On 
the other hand, given a $200 M_{\odot} \mathrm{yr}^{-1}$ star formation rate, the estimated emission from X-ray binaries, following

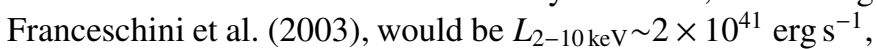
which is very close to the observed value $\left(\sim 3 \times 10^{41} \mathrm{erg} \mathrm{s}^{-1}\right)$. Xray spectra from supernovae have shown evidence for a strong Fe K line at $6.7 \mathrm{keV}$ (e.g. SN1986J, Houck et al. 1998), therefore could also account for this feature, while emission from $\mathrm{X}$-ray binaries cannot.

\section{Conclusions}

We analyzed the X-ray spectral properties of Mrk 273 combining the high throughput of XMM-Newton with the high spatial resolution of Chandra.

From the XMM-Newton spectrum we found a broad Fe $\mathrm{K} \alpha$ emission line with a high statistical significance ( $\gtrsim 99 \%$ c.l.). We suggested the superposition of multiple unresolved iron line features: one from neutral iron at $6.4 \mathrm{keV}(E W \sim 170 \mathrm{eV})$ and one from a blend of Fe XXV at $6.7 \mathrm{keV}(E W \sim 120 \mathrm{eV})$ and Fe XXVI at $6.97 \mathrm{keV}(E W<85 \mathrm{eV})$.

We tested two different models capable of reproducing the possible ionized iron lines detected: one through thermal emission, therefore involving the presence of a hot gas associated with a starburst, and the other one through reflection of the primary continuum by optically thin photoionized gas. Unfortunately, given the available statistics, we cannot rule out either of the two possible explanations. Some indications that a reflection componet is indeed present in the nuclear region of Mrk 273 may come from polarization measures, which are already available in the IR (Siebenmorgen \& Efstathiou 2001), but still not in the X-rays. High resolution spectroscopy could be helpful to detect some of the forbidden lines or the Radiative Recombination Continua (RRC), which are considered as typical signatures of photoionization (Porquet \& Dubau 2000; Liedahl 1999; Liedahl \& Paerels 1996).

Acknowledgements. We thank Günther Hasinger and Andrea Comastri for useful discussions, Stefano Bianchi and Paolo Tozzi for their help on the reduction and spectral analysis of the XMM-Newton and Chandra data and the anonymous referee for valuable comments and suggestions. This paper is based on observations obtained with XMM-Newton, an ESA science mission with instruments and contributions directly founded by ESA Member States and NASA.

\section{Appendix A: The unabsorbed Seyfert 2 Mrk 273x}

Over recent years, much attention has been directed to the study of unabsorbed Seyfert 2 galaxies (e.g. Ptak et al. 1996; Panessa $\&$ Bassani 2002; Gallo et al. 2005). These galaxies are of interest for two reason: they may account for as much as $30 \%$ of the Seyfert 2 population (Panessa \& Bassani 2002), and they are a challenge to explain with the existing Seyfert unification model.

Mrk 273x $(z=0.458)$ is one of the better known unabsorbed Seyfert 2 galaxies, perhaps because it falls in the fieldof-view of most observations of Mrk 273. In this appendix, we discuss the properties of Mrk $273 \mathrm{x}$ as seen during the XMM-Newton observation of Mrk 273.

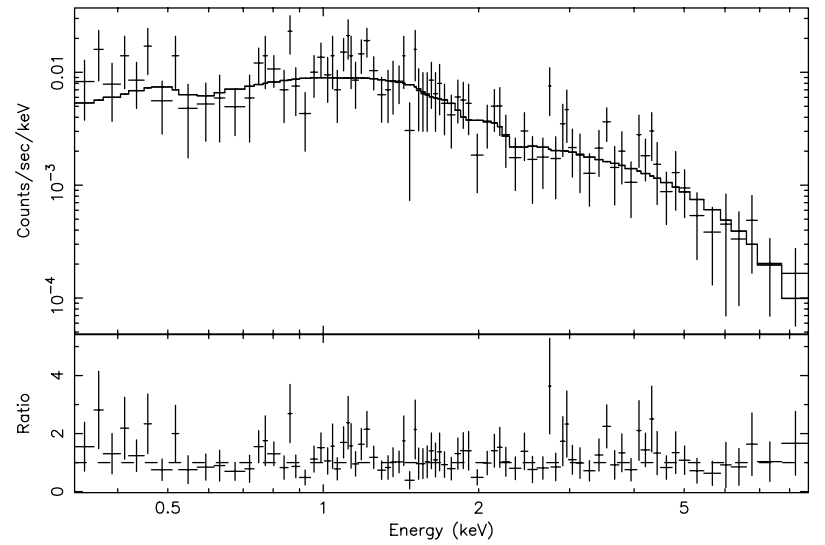

Fig. A.1. The combined MOS 1 and MOS2 $0.3-10 \mathrm{keV}$ data fitted with a power law modified by Galactic absorption.

In the EPIC-PN image, Mrk 273x falls directly in a chip gap; thus we used the combined MOS $1 / 2$ data for spectral analysis. The combined spectrum is source dominated in the $0.3-10 \mathrm{keV}$ band $(0.44-14.6 \mathrm{keV}$ in the rest-frame). The total number of source plus background counts is 526 , whereas the total number of background counts is 65 (scaled to source cell size).

The best-fit continuum model of Paper I to the Chandra data of Mrk 273x was a power law plus a weak thermal component. An emission line at $1.82 \mathrm{keV}$, which the authors suggested could be due to Si XVI, was significantly detected above the continuum. The XMM-Newton data are consistent with a power law $(\Gamma=1.49 \pm 0.12)$ modified by Galactic absorption $\left(\chi^{2} /\right.$ d.o.f. $=60.2 / 82$; Fig. A. 1$)$.

The $90 \%$ confidence upper limit on the intrinsic absorption is $<4.5 \times 10^{20} \mathrm{~cm}^{-2}$, at least a factor of three less than what was required in the Chandra model.

Multi-component continuum models (e.g. blackbody plus power law, double power law, or a broken power law) also fit the data well, but they are not significant improvements over the single power law. The addition of a $\sim 1.82 \mathrm{keV}$ emission feature is not required by the data $\left(\Delta \chi^{2}=1\right.$ for two additional free parameters; $E W<33 \mathrm{eV}$ ). Positive residuals at about $4.3 \mathrm{keV}$ can be modelled with a narrow Gaussian profile with a rest-frame energy of $E=6.29_{-0.34}^{+0.14} \mathrm{keV}$. The addition of the Gaussian profile is an improvement to the residuals, but it is not statistically significant $\left(\Delta \chi^{2}=3\right.$ for three additional free parameters).

Based on the single power law fit, the unabsorbed $0.5-10 \mathrm{keV}$ flux is $\left(1.43_{-0.18}^{+0.22}\right) \times 10^{-13} \mathrm{erg} \mathrm{s}^{-1} \mathrm{~cm}^{-2}(1.18 \times$ $10^{-13} \mathrm{erg} \mathrm{s}^{-1} \mathrm{~cm}^{-2}$ in the $2-10 \mathrm{keV}$ band). The intrinsic $0.3-10 \mathrm{keV}$ unabsorbed luminosity is $9.2 \times 10^{43} \mathrm{erg} \mathrm{s}^{-1}$. The broad band flux measured during this XMM-Newton observation is comparable to the flux observed during the Chandra observation two years earlier. The $\sim 22 \mathrm{ks}$ light curve of Mrk $273 \mathrm{x}$ shows no variability and is perfectly fitted by a constant.

\section{References}

Anders, E., \& Grevesse, N. 1989, Geochim. Cosmochim. Acta, 53, 197

Bianchi, S., \& Matt, G. 2002, A\&A, 387, 76 
Bianchi, S., Miniutti, G., Fabian, A. C., \& Iwasawa, K. 2005, MNRAS, 426

Boller, T., Keil, R., Hasinger, G., et al. 2003, A\&A, 411, 63

Bondi, M., Perez-Torres, M.-A., Dallacasa, D., \& Muxlow, T. 2005, MNRAS, Accepted [arXiv: astro-ph/0505370]

Braito, V., Franceschini, A., Della Ceca, R., et al. 2003, A\&A, 398, 107

Brandt, W. N., Fabian, A. C., Takahashi, K., et al. 1997, MNRAS, 290, 617

Carilli, C. L., \& Taylor, G. B. 2000, ApJ, 532, L95

den Herder, J. W., Brinkman, A. C., Kahn, S. M., et al. 2001, A\&A, 365, L7

Done, C., Madejski, G. M., Życki, P. T., \& Greenhill, L. J. 2003, ApJ, 588,763

Fox, D. W., Lewin, W. H. G., Fabian, A., et al. 2000, MNRAS, 319, 1154

Franceschini, A., Braito, V., Persic, M., et al. 2003, MNRAS, 343, 1181

Gallo, L., Lehmann, I., Pietsch, W., et al. 2005, MNRAS, submitted

Genzel, R., Lutz, D., Sturm, E., et al. 1998, ApJ, 498, 579

Goldader, J. D., Joseph, R. D., Doyon, R., \& Sanders, D. B. 1995 , ApJ, 444, 97

Houck, J. C., Bregman, J. N., Chevalier, R. A., \& Tomisaka, K. 1998, ApJ, 493, 431

Iwasawa, K. 1999, MNRAS, 302, 96

Iwasawa, K., Sanders, D. B., Evans, A. S., et al. 2005, MNRAS, 357, 565

Jansen, F., Lumb, D., Altieri, B., et al. 2001, A\&A, 365, L1

Kennicutt, R. C. 1998, ARA\&A, 36, 189

Kii, T., Nakagawa, T., Fujimoto, R., et al. 1997, in X-Ray Imaging and Spectroscopy of Cosmic Hot Plasmas, 161

Kinkhabwala, A., Sako, M., Behar, E., et al. 2002, ApJ, 575, 732

Knapen, J. H., Laine, S., Yates, J. A., et al. 1997, ApJ, 490, L29

Koski, A. T. 1978, ApJ, 223, 56

Liedahl, D. A. 1999, X-Ray Spectroscopy in Astrophysics, LNP, 520, 189

Liedahl, D. A., \& Paerels, F. 1996, ApJ, 468, L33

Lutz, D., Spoon, H. W. W., Rigopoulou, D., Moorwood, A. F. M., \& Genzel, R. 1998, ApJ, 505, L103

Mason, K. O., Breeveld, A., Much, R., et al. 2001, A\&A, 365, L36
Mitsuda, K. 1995, in Seventeeth Texas Symposium on Relativistic Astrophysics and Cosmology, 213

Netzer, H., Lemze, D., Kaspi, S., et al. 2005, ApJ in press [arXiv: astro-ph/0505016]

Panessa, F., \& Bassani, L. 2002, A\&A, 394, 435

Persic, M., \& Rephaeli, Y. 2002, A\&A, 382, 843

Pietsch, W., Roberts, T. P., Sako, M., et al. 2001, A\&A, 365, L174

Porquet, D., \& Dubau, J. 2000, A\&AS, 143, 495

Ptak, A., Yaqoob, T., Serlemitsos, P. J., Kunieda, H., \& Terashima, Y. 1996, ApJ, 459, 542

Ptak, A., Heckman, T., Levenson, N. A., Weaver, K., \& Strickland, D. 2003, ApJ, 592, 782

Risaliti, G., Gilli, R., Maiolino, R., \& Salvati, M. 2000, A\&A, 357, 13

Ruiz, M., Efstathiou, A., Alexander, D. M., \& Hough, J. 2001, MNRAS, 325, 995

Sako, M., Kahn, S. M., Paerels, F., \& Liedahl, D. A. 2000, ApJ, 543, L115

Sambruna, R. M., Netzer, H., Kaspi, S., et al. 2001, ApJ, 546, L13

Sanders, D. B., \& Mirabel, I. F. 1996, ARA\&A, 34, 749

Sanders, D. B., Soifer, B. T., Elias, J. H., et al. 1988a, ApJ, 325, 74

Sanders, D. B., Soifer, B. T., Elias, J. H., Neugebauer, G., \& Matthews, K. 1988b, ApJ, 328, L35

Sanders, D. B., Surace, J. A., \& Ishida, C. M. 1999, in Galaxy Interactions at Low and High Redshift, IAU Symp., 186, 289

Siebenmorgen, R., \& Efstathiou, A. 2001, A\&A, 376, L35

Smith, D. A., \& Wilson, A. S. 2003, ApJ, 591, 138

Smith, R. K., Brickhouse, N. S., Liedahl, D. A., \& Raymond, J. C. 2001, ApJ, 556, L91

Soifer, B. T., Sanders, D. B., Madore, B. F., et al. 1987, ApJ, 320, 238

Soifer, B. T., Neugebauer, G., Matthews, K., et al. 2000, AJ, 119, 509

Strüder, L., Briel, U., Dennerl, K., et al. 2001, A\&A, 365, L18

Surace, J. A., \& Sanders, D. B. 2000, AJ, 120, 604

Turner, T. J., George, I. M., Nandra, K., \& Mushotzky, R. F. 1997, ApJS, 113, 23

Turner, T. J., George, I. M., Nandra, K., \& Mushotzky, R. F. 1998, ApJ, 493, 91

Turner, M. J. L., Abbey, A., Arnaud, M., et al. 2001, A\&A, 365, L27

Vignati, P., Molendi, S., Matt, G., et al. 1999, A\&A, 349, L57

Xia, X. Y., Xue, S. J., Mao, S., et al. 2002, ApJ, 564, 196

Young, A. J., Wilson, A. S., \& Shopbell, P. L. 2001, ApJ, 556, 6 